\title{
The Applications of Algebraic Methods on Stable Analysis for General Differential Dynamical Systems with Multidelays
}

\author{
Jian $\mathrm{Ma}^{1}$ and Baodong Zheng ${ }^{2}$ \\ ${ }^{1}$ Department of Mathematics, Northeast Forestry University, Harbin 150040, China \\ ${ }^{2}$ Department of Mathematics, Harbin Institute of Technology, Harbin 150001, China \\ Correspondence should be addressed to Jian Ma; mj@nefu.edu.cn
}

Received 13 January 2015; Revised 31 March 2015; Accepted 3 April 2015

Academic Editor: Peng Shi

Copyright (c) $2015 \mathrm{~J}$. Ma and B. Zheng. This is an open access article distributed under the Creative Commons Attribution License, which permits unrestricted use, distribution, and reproduction in any medium, provided the original work is properly cited.

\begin{abstract}
The distribution of purely imaginary eigenvalues and stabilities of generally singular or neutral differential dynamical systems with multidelays are discussed. Choosing delays as parameters, firstly with commensurate case, we find new algebraic criteria to determine the distribution of purely imaginary eigenvalues by using matrix pencil, linear operator, matrix polynomial eigenvalues problem, and the Kronecker product. Additionally, we get practical checkable conditions to verdict the asymptotic stability and Hopf bifurcation of differential dynamical systems. At last, with more general case, the incommensurate, we mainly study critical delays when the system appears purely imaginary eigenvalue.
\end{abstract}

\section{Introduction}

Functional differential systems with multiple delays are important mathematic models to describe all kinds of natural and society phenomena. So it is used in many fields, such as lossless transmission lines, partial element equivalent circuits in electrical engineering, combustion systems, and controlled constrained manipulators in mechanical engineering. The asymptotic stabilization of differential systems with multiple delays is an important property in many applications. In the past decades, many results have been derived. The bifurcations and the stability analysis of functional differential systems with delays especially have received much attention by researchers and many excellent results have been obtained; see [1-7]. The asymptotic stability of differential systems with multiple delays can be established from the rightmost part of the spectrum. In the previous paper [8], we discussed a singular neutral linear differential system with a single delay. We now consider more general classes of differential system with multiple delays, that is, the general neutral linear differential system with multiple delays:

$$
\begin{aligned}
B_{0} \dot{X}(t)+\sum_{k=1}^{m} B_{k} \dot{X}\left(t-\tau_{k}\right) \\
=A_{0} X(t)+\sum_{k=1}^{m} A_{k} X\left(t-\tau_{k}\right),
\end{aligned}
$$

where coefficient matrix $A_{k}, B_{k} \in \mathbb{R}^{n \times n}(k=0,1, \ldots, m)$. $t(t \geq 0)$ is a time variant. $\tau_{k}\left(\tau_{k} \geq 0, k=1,2, \ldots, m\right)$ denote the delayed parameter, which are ordered increasingly; that is, $\tau_{1}<\tau_{2}<\cdots<\tau_{m} . X(t)\left(X(t)=\left(x_{1}(t), x_{2}(t), \ldots, x_{n}(t)\right)^{T}\right)$ is a state variant, which is given by continuous functions on the initial interval $\left[-\tau_{m}, 0\right]$; that is,

$$
X(t)=\Phi(t), \quad t \in\left[-\tau_{m}, 0\right] .
$$

Notice that (1) is quite general, which contains many subclasses. For example, when the leading matrix $B_{0}$ satisfies Rank $B_{0}<n$, the system is called a singular neutral delayed differential system, which is also called a delayed differentialalgebraic system. Besides, when Rank $B_{0}=n$, a special subclass of (1) is written as

$$
\dot{X}(t)+\sum_{k=1}^{m} B_{k} \dot{X}\left(t-\tau_{k}\right)=A_{0} X(t)+\sum_{k=1}^{m} A_{k} X\left(t-\tau_{k}\right),
$$

which is a nonsingular neutral delayed differential system. For (1) especially, if coefficient matrix $B_{k}=0(k=1,2$, $\ldots, m)$, we have the singular retarded differential system

$$
B_{0} \dot{X}(t)=A_{0} X(t)+\sum_{k=1}^{m} A_{k} X\left(t-\tau_{k}\right) .
$$


At last, if $\operatorname{Rank} B_{0}=n$, we have the nonsingular retarded delay-differential system

$$
\dot{X}(t)=A_{0} X(t)+\sum_{k=1}^{m} A_{k} X\left(t-\tau_{k}\right) .
$$

For (4) and (5), dynamic behaviors have been well studied by many researchers, and the theory of stability has been well known and discussed for decades in a wide range of literature; see [9-15]. But for linear neutral delayed system (4) or (5), the necessary and sufficient stability conditions are scarce and less efficient than their counterparts for retarded systems (1) and (3). Besides, for the derived results, mainly researched methods can be divided into two cases, analytic methods and numerical methods. The analytic methods contain $V$-functional methods, Laplace transformation, central manifolds, normal forms, and so on. The numerical methods mainly contain linear multistep methods, RungeKutta methods, Newton methods, $\theta$-methods, and so on. Those methods are main keys to solve problems of stability on functional differential equations with delays all the time. By the development of delayed systems, many new methods appeared. In all of them, algebraic methods gradually grow up and have become a new and effective tool. For research on more complex systems especially, such as $n$-dimensional systems, algebraic methods are important tools to simplify the forms of time-delay systems. Certainly some results have been derived, yet there are more different time-delay systems waiting for study.

In this paper, we mainly discuss neutral delayed differential systems by algebraic methods. The main work is aimed at developing more efficient stability tests for neutral delay-differential systems. Compared to the retarded case, the neutral case induces complications. It is well known that for the retarded differential systems, number of eigenvalues in the right half plane is always finite. But for neutral differential systems, there exist some characteristic chains, when imaginary parts tend to infinity, real parts may have finite limit. That is to say, for some given characteristic chains $\left\{s_{n}\right\}$,

$$
\lim _{n \rightarrow+\infty} \operatorname{Re} s_{n}=a, \quad \text { if } \lim _{n \rightarrow+\infty} \operatorname{Im} s_{n}=\infty .
$$

In addition, spectrum of neutral differential systems may be sensitive to delay parameter changes, which exhibits some discontinuity. Even though each eigenvalue path is continuous, an infinitesimal change on delays may also cause the stability of system shifting. This discontinuity is closly related to the essential spectrum of system (1), that is, the spectrum of difference equation

$$
B_{0} X(t)+\sum_{k=1}^{m} B_{k} X\left(t-\tau_{k}\right)=0 .
$$

So the stable analysis on neutral differential systems often exhibits more complicated. In this paper, we will find criteria to find delays margin, in which the neutral system (1) or (3) is asymptotically stable or unstable.

The main contents in the following sections can be summarized as follows. In Section 2, we will introduce stable notions and present a number of preliminary facts. In Section 3, we will discuss the special case that delayed parameters are commensurate. By algebraic methods, such as matrix pencil, linear operators, and Kronecker product, delayed margin and stability of the neutral linear differential system (1) are derived. In Section 4, we will research the general case, that is, the neutral linear differential system with incommensurate delays. For both types of above systems, we derive the criteria of stability and the distribution of eigenvalues or generalized eigenvalues of constant matrix pencil. At last, this paper concludes in Section 5.

\section{Preliminary}

In this section, we begin with the description of main notation. Generally, let $\mathbb{R}$ denote the set of real numbers, $\mathbb{C}$ the set of complex numbers, and $\mathbb{R}^{+}$the set of nonnegative real numbers. Besides, we denote the open left half plane of the complex by $\mathbb{C}^{-}=\{s \mid \operatorname{Re}(s)<0\}$, the imaginary axis by $\partial \mathbb{C}$, and the open circle by $\partial D$. From [8], we know that the solvability of system (1) is determined by the regularity of matrix pencil $\left(B_{0}, A_{0}\right)$, which is regular if $s B_{0}-A_{0}$ is not identically singular for any complex $s$. Meantime, the zero $s$ of equation $\operatorname{det}\left(s B_{0}-A_{0}\right)=0$ is called the general eigenvalue of matrix pencil $\left(B_{0}, A_{0}\right)$. So in this paper, we suppose that the matrix pencil $\left(B_{0}, A_{0}\right)$ is always regular. In addition, the characteristic equation of system (1) is

$$
P(s) v=[-s B(s)+A(s)] v=0, \quad\|v\|=1,
$$

where

$$
\begin{aligned}
& B(s)=B_{0}+\sum_{k=1}^{m} B_{k} e^{-\tau_{k} s}, \\
& A(s)=A_{0}+\sum_{k=1}^{m} A_{k} e^{-\tau_{k} s} .
\end{aligned}
$$

The spectrum of system (1) is denoted by

$$
\sigma(P)=\{s \in \mathbb{C} \mid \operatorname{det}(P(s))=0\} .
$$

For the difference equation (7), its characteristic equation is

$$
Q(s) v=\left[B_{0}(s)+\sum_{k=1}^{m} B_{k} e^{-\tau_{k} s}\right] v=0, \quad\|v\|=1 .
$$

The spectrum of (7) is denoted by

$$
\sigma(Q)=\{s \in \mathbb{C} \mid \operatorname{det}(Q(s))=0\},
$$

which is also called the essential spectrum of system (1).

From the introduction, we know that neutral differential systems have many unpleasant properties, which have been specifically presented in $[16,17]$. Firstly, the real part of spectrum can have finite clustering points. Even the origin can be a clustering point. In fact, clustering points are contained in the closure of $\Delta(Q)$ :

$$
\Delta(Q)=\{\operatorname{Re} s \mid s \in \sigma(Q)\} .
$$


So the real part of the spectrum is not always continuously related to parameter delays. This discontinuity is clearly related to the neutral part of system (1). Certainly, for system (1), unlike retarded differential systems, it is not sufficient for stability that the spectrum is absolutely contained in the open left half plane of the complex plane. Fortunately, there is theory available in the literature which gives sufficient conditions for these pessimistic properties. By [6], we have derived that system (1) is asymptotically stable when

$$
\operatorname{Re} s \leq-\delta<0
$$

where $s$ is an eigenvalue and $\delta>0$ is a real number. The necessary condition on the stability of system (1) especially is that the difference equation (7) of neutral part of system (1) is stable, that is, the spectrum radius $\rho(Q)<1$. From [8], we all know that condition $\operatorname{Re} s \leq-\delta<0$ can be improved to $\operatorname{Re} s<0$ if the neutral part of system (1) is stable. That is to say, the critical condition for stability switch of system (1) is that the rightmost eigenvalue goes from the left half plane of the complex plane into the right half plane by passing the imaginary axis. So appearance of imaginary eigenvalues is a critical condition. In the following we will compute critical value of delayed parameters such that the stability switch occurs.

Next we will discuss the stability of system (1) by two sections. In Section 3, the delays are commensurate, in which phenomena appeared in many natural species. In Section 4, the delays are incommensurate. These dynamics have not been well understood yet. By algebraic methods, we can demonstrate the dynamical property more compactly and intuitionally.

\section{The Commensurate Case}

In this section, we will consider the neutral differential system with commensurate delays

$$
\begin{aligned}
& B_{0} \dot{X}(t)+\sum_{k=1}^{m} B_{k} \dot{X}(t-k \tau) \\
& =A_{0} X(t)+\sum_{k=1}^{m} A_{k} X(t-k \tau),
\end{aligned}
$$

where matrix $A_{k}, B_{k} \in \mathbb{R}^{n \times n}(k=0,1, \ldots, m)$. When $\operatorname{Rank} B_{0}<n$, system (15) is singular. When $\operatorname{Rank} B_{0}=n$, it can be rewritten as

$$
\begin{aligned}
& \dot{X}(t)+\sum_{k=1}^{m} B_{k} \dot{X}(t-k \tau) \\
& =A_{0} X(t)+\sum_{k=1}^{m} A_{k} X(t-k \tau),
\end{aligned}
$$

which is nonsingular. For system (15) and (16), some scholars have widely researched the delay-independent or delaydependent stability and asymptotic stability by analytic methods or numerical methods. Because of the complex nature of singular differential systems with delays, research is very difficult by using the analytical treatment. So few studies on stability and bifurcations have been conducted so far. For the singular neutral differential system (15) especially, there are hardly flexible and efficient verdicts. In the following, we will find algebraic criteria of the distribution of imaginary eigenvalues and stability for system (15).

3.1. Criteria for Determining Imaginary Eigenvalues. Firstly, an ordinary differential equation is considered, which motivates future research. Consider matrix equation as follows:

$$
\begin{gathered}
B \dot{X}(t)=A X(t), \\
\dot{X}^{T}(t) \bar{B}^{T}=-X^{T}(t) \bar{A}^{T},
\end{gathered}
$$

where $B=\left(B_{0}, B_{1}, \ldots, B_{m}\right), A=\left(A_{0}, A_{1}, \ldots, A_{m}\right), \bar{B}=\left(B_{m}\right.$, $\left.B_{m-1}, \ldots, B_{0}\right), \bar{A}=\left(A_{m}, A_{m-1}, \ldots, A_{0}\right), B_{k}, A_{k} \in \mathbb{R}^{n \times n}$, $X(t)=\left(X_{0}(t), X_{1}(t), \ldots, X_{m}(t)\right)^{T}, X_{k}(t) \in \mathbb{C}^{n \times n}, k=0,1$, $\ldots, m$. Let $V$ denote a vector space,

$$
V=\mathbb{C}^{((m+1) n) \times n} \times \mathbb{C}^{n \times((m+1) n)} .
$$

$L_{1}, L_{2}$ denote operators on $V$,

$$
\begin{gathered}
L_{1} X(t)=\left(\begin{array}{c}
B X(t) \\
X^{T}(t) \bar{B}^{T}
\end{array}\right), \\
L_{2} X(t)=\left(\begin{array}{c}
A X(t) \\
-X^{T}(t) \bar{A}^{T}
\end{array}\right), \\
\forall X(t) \in \mathbb{C}^{((m+1) n) \times n} .
\end{gathered}
$$

Then system (17) can be rewritten as

$$
L_{1} \dot{X}(t)=L_{2} X(t)
$$

Supposing $\widetilde{X}(t)=\left(Y e^{s t}, Y e^{s(t-\tau)}, Y e^{s(t-2 \tau)}, \ldots, Y e^{s(t-m \tau)}\right)^{T}$ is a matrix solution of system (17), we have

$$
\begin{array}{r}
{\left[\left(s \sum_{k=0}^{m} B_{k} z^{k}\right)-\left(\sum_{k=0}^{m} A_{k} z^{k}\right)\right] Y=0,} \\
Y^{T}\left[\left(s \sum_{k=0}^{m} B_{m-k}^{T} z^{k}\right)+\left(\sum_{k=0}^{m} A_{m-k}^{T} z^{k}\right)\right]=0,
\end{array}
$$


where $z=e^{-s \tau}$. For $\forall s \in \mathbb{C}, \mathbf{T}=\mathbf{T}(s), \Lambda=\Lambda(s)$ denote operators on $V$,

$$
\begin{aligned}
& \mathbf{T} X=\left(\left[\begin{array}{c}
\left.\left[s \sum_{k=0}^{m} B_{k} z^{k}\right)-\left(\sum_{k=0}^{m} A_{k} z^{k}\right)\right] X \\
X^{T}\left[\left(s \sum_{k=0}^{m} B_{m-k}^{T} z^{k}\right)+\left(\sum_{k=0}^{m} A_{m-k}^{T} z^{k}\right)\right]
\end{array}\right),\right. \\
& \forall X \in \mathbb{C}^{((m+1) n) \times n}, \\
& \Lambda X= \\
&+\left(\sum_{k=0}^{m} A_{k} z^{k}\right) X\left(\sum_{k=0}^{m} B_{m-k}^{T} z^{k}\right) \\
&+\left(\sum_{k=0}^{m} B_{k} z^{k}\right) X\left(\sum_{k=0}^{m} A_{m-k}^{T} z^{k}\right), \\
& \forall X \in \mathbb{C}^{((m+1) n) \times n} .
\end{aligned}
$$

We have $T Y=0$; that is,

$$
\begin{aligned}
\Lambda \cdot Y Y^{T}= & \left(\sum_{k=0}^{m} A_{k} z^{k}\right) Y Y^{T}\left(\sum_{k=0}^{m} B_{m-k}^{T} z^{k}\right) \\
& +\left(\sum_{k=0}^{m} B_{k} z^{k}\right) Y Y^{T}\left(\sum_{k=0}^{m} A_{m-k}^{T} z^{k}\right)=0 .
\end{aligned}
$$

By the property of Kronecker product, we have

$$
\begin{gathered}
{\left[\left(\sum_{k=0}^{m} A_{k} z^{k}\right) \otimes\left(\sum_{k=0}^{m} B_{m-k} z^{k}\right)+\left(\sum_{k=0}^{m} B_{k} z^{k}\right)\right.} \\
\left.\otimes\left(\sum_{k=0}^{m} A_{m-k} z^{k}\right)\right] \cdot \xi\left(Y Y^{T}\right)=0 .
\end{gathered}
$$

So

$$
\begin{aligned}
& \operatorname{det}\left[\left(\sum_{k=0}^{m} A_{k} z^{k}\right) \otimes\left(\sum_{k=0}^{m} B_{m-k} z^{k}\right)+\left(\sum_{k=0}^{m} B_{k} z^{k}\right)\right. \\
& \left.\otimes\left(\sum_{k=0}^{m} A_{m-k} z^{k}\right)\right]=0 .
\end{aligned}
$$

Theorem 1. Any imaginary eigenvalue of system (15) is one of roots of (25).

Proof. The characteristic equation of system (15) is

$$
p(s, z) v=\left[-s \sum_{k=0}^{m} B_{k} z^{k}+\sum_{k=0}^{m} A_{k} z^{k}\right] v=0, \quad\|v\|=1,
$$

where $z=e^{-\tau s}$. Suppose $s=i w$; by conjugating and transforming we have

$$
v^{T}\left[s \sum_{k=0}^{m} B_{m-k}^{T} z^{k}+\sum_{k=0}^{m} A_{m-k}^{T} z^{k}\right]=0 .
$$

By (26) and (27),

$$
\begin{gathered}
s \sum_{k=0}^{m} B_{k} z^{k} v=\sum_{k=0}^{m} A_{k} z^{k} v, \\
-s v^{T} \sum_{k=0}^{m} B_{m-k}^{T} z^{k}=v^{T} \sum_{k=0}^{m} A_{m-k}^{T} z^{k} .
\end{gathered}
$$

By simplistically computing,

$$
\begin{gathered}
s\left[\sum_{k=0}^{m} B_{k} z^{k}\right] v v^{T}\left[\sum_{k=0}^{m} B_{m-k}^{T} z^{k}\right] \\
=\left[\sum_{k=0}^{m} A_{k} z^{k}\right] v v^{T}\left[\sum_{k=0}^{m} B_{m-k}^{T} z^{k}\right], \\
-s\left[\sum_{k=0}^{m} B_{k} z^{k}\right] v v^{T}\left[\sum_{k=0}^{m} B_{m-k}^{T} z^{k}\right] \\
=\left[\sum_{k=0}^{m} B_{k} z^{k}\right] v v^{T}\left[\sum_{k=0}^{m} A_{m-k}^{T} z^{k}\right] .
\end{gathered}
$$

So

$$
\begin{aligned}
{\left[\sum_{k=0}^{m} A_{k} z^{k}\right] v v^{T}\left[\sum_{k=0}^{m} B_{m-k}^{T} z^{k}\right] } \\
+\left[\sum_{k=0}^{m} B_{k} z^{k}\right] v v^{T}\left[\sum_{k=0}^{m} A_{m-k}^{T} z^{k}\right]=0 .
\end{aligned}
$$

By above operator, the result can be derived.

From [18], (25) is a polynomial eigenvalue problem. By linearization, we have the following lemma.

Lemma 2. All of $z=e^{-s \tau}$ of the characteristic equation of system (15) are general eigenvalues of the matrix pencil $(E, F)$, where

$$
\begin{aligned}
E & =\left(\begin{array}{cccc}
C_{2 m} & 0 & \cdots & 0 \\
0 & I_{n} & \cdots & 0 \\
\vdots & \vdots & \ddots & \vdots \\
0 & 0 & \cdots & I_{n}
\end{array}\right), \\
F & =\left(\begin{array}{ccccc}
-C_{2 m-1} & -C_{2 m-2} & \cdots & -C_{1} & -C_{0} \\
I_{n} & 0 & \cdots & 0 & 0 \\
0 & I_{n} & \cdots & 0 & 0 \\
\vdots & \vdots & \ddots & \vdots & \vdots \\
0 & 0 & \cdots & I_{n} & 0
\end{array}\right) .
\end{aligned}
$$


Proof. By (25) and property of Kronecker product, we have

$$
\begin{aligned}
& \operatorname{det}\left[A_{0} \otimes B_{m}+B_{0} \otimes A_{m}+\sum_{k=1}^{m} z^{k}\left(A_{0} \otimes B_{m-k}+A_{K}\right.\right. \\
& \left.\otimes B_{m}+B_{0} \otimes A_{m-k}+B_{K} \otimes A_{m}\right)+\sum_{k=1, j=1}^{m} z^{k+j}\left(A_{k}\right. \\
& \left.\left.\otimes B_{m-j}+B_{K} \otimes A_{m-j}\right)\right]=0,
\end{aligned}
$$

which is a polynomial equation with $2 m$ degrees about variant $z$

$$
\begin{aligned}
\operatorname{det} & {\left[P_{2 m}(z)\right] } \\
= & \operatorname{det}\left[C_{2 m} z^{2 m}+C_{2 m-1} z^{2 m-1}+\cdots+C_{1} z+C_{0}\right] \\
= & 0 ;
\end{aligned}
$$

that is

$$
P_{2 m}(z) Y=0, \quad Y=\xi\left(v v^{T}\right)
$$

Let

$$
U=\left(\begin{array}{c}
u_{1} \\
u_{2} \\
\vdots \\
u_{2 m}
\end{array}\right)=\left(\begin{array}{c}
z^{2 m-1} Y \\
z^{2 m-2} Y \\
\vdots \\
z Y \\
Y
\end{array}\right)
$$

We can get

$$
\begin{aligned}
P_{2 m}(z) Y= & C_{2 m} z z^{2 m-1} Y+C_{2 m-1} z^{2 m-1} Y+\cdots \\
& +C_{1} z Y+C_{0} Y
\end{aligned}
$$

which can be written as

$$
\begin{aligned}
P_{2 m}(z) Y= & C_{2 m} z z^{2 m-1} Y+C_{2 m-1} z^{2 m-1} Y+\cdots \\
& +C_{1} z Y+C_{0} Y .
\end{aligned}
$$

By the companion form, the linearization of (25) is

$$
P_{2 m}(z) U=[z E-F] U=0 .
$$

So $z$ is the general eigenvalue of matrix pencil $(E, F)$.

3.2. Criteria for the Asymptotic Stability. Stability of delaydifferential equations has been widely researched [19-21]. We all know that the Lyapunov-Kraeovskii functional approach is an important analytic method to discuss delay-independent stability. Results for singular neutral differential systems are still very few, especially by algebraic methods. Next we first research the delay-independent stability of system (15).
Theorem 3. If coefficient matrices of system (15) satisfy the following,

(1) for matrix pencil $\left(-B_{0}, A_{0}\right), \sigma\left(-B_{0}, A_{0}\right) \in C^{-}$,

(2) $\sup _{\operatorname{Re}(s)=0} \rho\left(\sum_{i=1}^{m}\left|\left(-s B_{0}+A_{0}\right)^{-1}\left(-s B_{i}+A_{i}\right)\right|\right)<1$,

then the stability of system (15) is delay-independent; that is, system (15) is asymptotically stable for all $\tau \geq 0$.

Proof. See [2].

Besides, it is well known that if the neutral part of system (15) is stable, then system (15) is also stable when all eigenvalues pose in the half plane of the complex plane. For the difference equation:

$$
\sum_{k=0}^{m} B_{k} X(t-k \tau)=0 .
$$

The characteristic equation is

$$
Q(s, z) v=\left(\sum_{k=0}^{m} B_{k} z^{k}\right) v=0, \quad\|v\|=1 .
$$

Lemma 4. Variant $z$ is contained in the spectrum of matrix pencil $(M, N)$, where

$$
\begin{aligned}
M & =\left(\begin{array}{cccc}
B_{m} & 0 & \cdots & 0 \\
0 & I_{n} & \cdots & 0 \\
\vdots & \vdots & \ddots & \vdots \\
0 & 0 & \cdots & I_{n}
\end{array}\right), \\
N & =\left(\begin{array}{ccccc}
-B_{m-1} & -B_{m-2} & \cdots & -B_{2} & -B_{1} \\
I_{n} & 0 & \cdots & 0 & 0 \\
0 & I_{n} & \cdots & 0 & 0 \\
\vdots & \vdots & \ddots & \vdots & \vdots \\
0 & 0 & \cdots & I_{n} & 0
\end{array}\right) .
\end{aligned}
$$

Proof. Comparing with Lemma 2, Let

$$
U=\left(\begin{array}{c}
v \\
z v \\
\vdots \\
z^{m-1} v
\end{array}\right)
$$

we can get $\sigma(M, N)=\{z \mid \operatorname{det}(z M-N)=0\}$.

Apparently, the difference equation is stable when spectrum radius $\rho(M, N)<1$. If this condition is satisfied, then system (15) is asymptotically stable when all eigenvalues have negative real part. So we have the following result.

Theorem 5. Suppose system (15) is stable at $\tau=0$ and $\rho(M, N)<1$. 
(1) If system (15) satisfies one of the following conditions,

$$
\begin{aligned}
& \text { (a) } \sigma(E, F) \cap \partial D=\emptyset, \\
& \text { (b) } \sigma(E, F) \cap \partial D \neq \emptyset \text {, for } z_{k} \in \sigma(E, F) \cap \partial D, \sigma(p(s, \\
& \left.\left.z_{k}\right)\right)=\{0\}, \\
& \text { (c) } \sigma(E, F) \cap \partial D \neq \emptyset, \forall z_{k} \in \sigma(E, F) \cap \partial D, \sigma(p(s, \\
& \left.\left.z_{k}\right)\right) \cap \partial C^{+}=\emptyset,
\end{aligned}
$$

then system (15) is asymptotically stable for $\tau \in[0$, $+\infty)$.

(2) Otherwise, if $\sigma\left(p\left(s, z_{k}\right)\right) \cap \partial C^{+} \neq \emptyset$, there is $s=i w_{k}^{(j)} \in$ $R^{+}, j=1,2, \ldots, t, t \leq l$. Let $z_{k}=e^{i \beta_{k}}$, which satisfies $\operatorname{det}\left[p\left(i w_{k}^{(j)}, z_{k}\right)\right]=0$. Then there exists

$$
\tau^{*}=\min _{1 \leq k \leq l} \min _{1 \leq j \leq t} \frac{\beta_{k}}{w_{k}^{(j)}} .
$$

System (15) is asymptotically stable at $\left[0, \tau^{*}\right)$ and unstable when $\tau>\tau^{*}$ and bifurcates at $\tau=\tau^{*}$.

\section{The Incommensurate Case}

For the general case, system (1) can be rewritten as

$$
\sum_{k=0}^{m} B_{k} \dot{X}\left(t-\tau_{k}\right)=\sum_{k=0}^{m} A_{k} X\left(t-\tau_{k}\right),
$$

where $\tau_{0}=0$. For the simplicity, the characteristic equation can also be rewritten as

$$
M(s) v=[-s B(s)+A(s)] v=0,
$$

where

$$
\begin{aligned}
& B(s)=\sum_{k=0}^{m} B_{k} e^{-s \tau_{k}}, \\
& A(s)=\sum_{k=0}^{m} A_{k} e^{-s \tau_{k}} .
\end{aligned}
$$

Apparently, the stability of system (44) is similar to system (15). Firstly, for the delay-independent stability, we have the same conclusion.

\section{Theorem 6. If}

(1) $\sigma\left(-B_{0}, A_{0}\right) \in C^{-}$,

(2) $\sup _{\operatorname{Re}(s)=0} \rho\left(\sum_{i=1}^{m}\left|\left(-s B_{0}+A_{0}\right)^{-1}\left(-s B_{i}+A_{i}\right)\right|\right)<1$,

then the stability of system (44) is delay-independent; that is, system (44) is asymptotically stable for delay parameters $\left\{\tau_{k}\right\}\left(\tau_{k}>0, k=1,2, \ldots, m\right)$.

Proof. See Theorem 3.

Next, we will focus on delay-dependent stability conditions. For delay parameters $\left\{\tau_{k}\right\}(k=1,2, \ldots, m)$, let $r\left(\tau_{1}, \tau_{2}, \ldots, \tau_{m}\right)$ denote the real part of the rightmost eigenvalue or the corresponding supremum. Unlike retarded delay-differential systems, system (44) is asymptotically stable if the real part of the spectrum lies in the left half plane of complex plane and is bounded away from a negative number; that is,

$$
r\left(\tau_{1}, \tau_{2}, \ldots, \tau_{m}\right)<-\delta<0 .
$$

The associated difference equation is

$$
\sum_{k=0}^{m} B_{k} X\left(t-\tau_{k}\right)=0
$$

As we all know if system (48) is strongly exponentially stable and the origin is not a clustering point of real part of spectrum, the spectral abscissa (the real part of eigenvalues) of system (44) will be continuous on delay parameters $\left\{\tau_{k}\right\}$. Under above condition, system (44) is asymptotically stable when

$$
r\left(\tau_{1}, \tau_{2}, \ldots, \tau_{m}\right)<0 .
$$

When $r\left(\tau_{1}, \tau_{2}, \ldots, \tau_{m}\right)>0$, the system (44) is unstable. So the stability of system (44) could shift when $r\left(\tau_{1}, \tau_{2}, \ldots, \tau_{m}\right)=$ 0 . The root $\left(\tau_{1}^{*}, \tau_{2}^{*}, \ldots, \tau_{m}^{*}\right)$ of $r\left(\tau_{1}, \tau_{2}, \ldots, \tau_{m}\right)=0$ is called critical delays. In the following, we will find algebraic criteria for the critical delays.

First, assuming that system (44) is hyperbolic (the spectrum has no zero eigenvalue) and zero is not a clustering point of the real part of spectrum. Let $s=i w, w \in \mathbb{R}$ be the purely imaginary eigenvalue and $v \in \mathbb{C}^{n}$ be the corresponding eigenvector. Meantime, let $\varphi_{k}=w \tau_{k}(k=0,1,2, \ldots, m-1)$ denote $m$ free parameters $\left(\varphi_{0}=0\right)$ and $z=e^{-i w \tau_{m}} \in \partial D$. For the characteristic equation (45), we have

$$
s\left[z B_{m}+\sum_{k=0}^{m-1} B_{k} e^{-i \varphi_{k}}\right] v=\left[z A_{m}+\sum_{k=0}^{m-1} A_{k} e^{-i \varphi_{k}}\right] v,
$$

where $A(\vec{\varphi})=\sum_{k=0}^{m-1} A_{k} e^{-i \varphi_{k}}, B(\vec{\varphi})=\sum_{k=0}^{m-1} B_{k} e^{-i \varphi_{k}}$. Then

$$
s\left[z B_{m}+B(\vec{\varphi})\right] v=\left[z A_{m}+A(\vec{\varphi})\right] v .
$$

By conjugating and transforming, we have

$$
-s v^{*}\left[\frac{1}{z} B_{m}^{*}+B^{*}(\vec{\varphi})\right]=v^{*}\left[\frac{1}{z} A_{m}^{*}+A^{*}(\vec{\varphi})\right] .
$$

Multiplying with $z$,

$$
-s v^{*}\left[B_{m}^{*}+z B^{*}(\vec{\varphi})\right]=v^{*}\left[A_{m}^{*}+z A^{*}(\vec{\varphi})\right] .
$$

That is

$$
\begin{aligned}
s & {\left[z B_{m}+B(\vec{\varphi})\right] v v^{*}\left[B_{m}^{*}+z B^{*}(\vec{\varphi})\right] } \\
& =\left[z A_{m}+A(\vec{\varphi})\right] v v^{*}\left[B_{m}^{*}+z B^{*}(\vec{\varphi})\right], \\
-s & {\left[z B_{m}+B(\vec{\varphi})\right] v v^{*}\left[B_{m}^{*}+z B^{*}(\vec{\varphi})\right] } \\
& =\left[z B_{m}+B(\vec{\varphi})\right] v v^{*}\left[A_{m}^{*}+z A^{*}(\vec{\varphi})\right],
\end{aligned}
$$


So

$$
\begin{aligned}
& {\left[z B_{m}+B(\vec{\varphi})\right] v v^{*}\left[A_{m}^{*}+z A^{*}(\vec{\varphi})\right]} \\
& \quad+\left[z A_{m}+A(\vec{\varphi})\right] v v^{*}\left[B_{m}^{*}+z B^{*}(\vec{\varphi})\right]=0 .
\end{aligned}
$$

Expanding (55)

$$
\begin{aligned}
z^{2} & {\left[B_{m} v v^{*} A^{*}(\vec{\varphi})+A_{m} v v^{*} B^{*}(\vec{\varphi})\right]+z\left[B_{m} v v^{*} A_{m}^{*}\right.} \\
& +B(\vec{\varphi}) v v^{*} A^{*}(\vec{\varphi})+A_{m} v v^{*} B_{m}^{*} \\
& \left.+A(\vec{\varphi}) v v^{*} B^{*}(\vec{\varphi})\right]+B(\vec{\varphi}) v v^{*} A_{m}^{*}+A(\vec{\varphi}) v v^{*} B_{m}^{*} \\
& =0 .
\end{aligned}
$$

By Kronecker property, we have

$$
\begin{aligned}
& {\left[z^{2}\left(B_{m} \otimes A(-\vec{\varphi})+A_{m} \otimes B(-\vec{\varphi})\right)+z\left(B_{m} \otimes A_{m}\right.\right.} \\
& \left.\quad+B(\vec{\varphi}) \otimes A(-\vec{\varphi})+A_{m} \otimes B_{m}+A(\vec{\varphi}) \otimes B(-\vec{\varphi})\right) \\
& \left.+B(\vec{\varphi}) \otimes A_{m}+A(\vec{\varphi}) \otimes B_{m}\right] \xi\left(v v^{*}\right)=0 .
\end{aligned}
$$

Let

$$
\begin{aligned}
X(\vec{\varphi})= & B_{m} \otimes A(-\vec{\varphi})+A_{m} \otimes B(-\vec{\varphi}), \\
Y(\vec{\varphi})= & B_{m} \otimes A_{m}+B(\vec{\varphi}) \otimes A(-\vec{\varphi})+A_{m} \otimes B_{m} \\
& +A(\vec{\varphi}) \otimes B(-\vec{\varphi}), \\
Z(\vec{\varphi})= & B(\vec{\varphi}) \otimes A_{m}+A(\vec{\varphi}) \otimes B_{m}, \\
u= & \xi\left(v v^{*}\right),
\end{aligned}
$$

so we have

$$
z^{2} X(\vec{\varphi})+z Y(\vec{\varphi})+Z(\vec{\varphi}) u=0 .
$$

Lemma 7. All of $z=e^{-s \tau_{m}}$ of the characteristic equation of system (44) are general eigenvalues of matrix pencil $(E, F)$, where

$$
\begin{aligned}
& E=\left(\begin{array}{cc}
X(\vec{\varphi}) & 0 \\
0 & I
\end{array}\right), \\
& F=\left(\begin{array}{cc}
-Y(\vec{\varphi}) & -Z(\vec{\varphi}) \\
I & 0
\end{array}\right) .
\end{aligned}
$$

Proof. The proof is similar to Lemma 2.

Theorem 8. Assuming that system (44) is hyperbolic and zero is not root of $B(s) v=0$, then critical delays satisfy

$$
\begin{aligned}
\tau_{k} & =\frac{\varphi_{k}+2 n_{k} \pi}{w}, \quad n_{k} \in \mathbb{Z}, k=1,2, \ldots, m-1, \\
\tau_{m} & =\frac{-\operatorname{Arg} z+2 n_{m} \pi}{w}, \quad n_{m} \in \mathbb{Z},
\end{aligned}
$$

where

$$
w=-i \frac{v^{*}\left[B_{m}^{*}+z B^{*}(\vec{\varphi})\right]\left[z A_{m}+A(\vec{\varphi})\right] v}{v^{*}\left[B_{m}^{*}+z B^{*}(\vec{\varphi})\right]\left[z B_{m}+B(\vec{\varphi})\right] v} \in \mathbb{R},
$$

$z \in \partial D$ is general eigenvalue of matrix pencil $(E, F), s=$ $i w(w \in \mathbb{R})$ is the purely imaginary eigenvalue, $v \in \mathbb{C}^{n}$, and $v \neq 0$ is the corresponding eigenvector.

Proof. For the characteristic equation of system (44)

$$
s\left[z B_{m}+B(\vec{\varphi})\right] v=\left[z A_{m}+A(\vec{\varphi})\right] v .
$$

By multiplying with $v^{*}\left[z B_{m}+B(\vec{\varphi})\right]^{*}$

$$
\begin{aligned}
s v^{*} & {\left[z B_{m}+B(\vec{\varphi})\right]^{*}\left[z B_{m}+B(\vec{\varphi})\right] v } \\
& =v^{*}\left[z B_{m}+B(\vec{\varphi})\right]^{*}\left[z A_{m}+A(\vec{\varphi})\right] v .
\end{aligned}
$$

Apparently, $v^{*}\left[z B_{m}+B(\vec{\varphi})\right]^{*}\left[z B_{m}+B(\vec{\varphi})\right] v \in \mathbb{R}$, so

$$
s=\frac{v^{*}\left[z B_{m}+B(\vec{\varphi})\right]^{*}\left[z A_{m}+A(\vec{\varphi})\right] v}{v^{*}\left[z B_{m}+B(\vec{\varphi})\right]^{*}\left[z B_{m}+B(\vec{\varphi})\right] v} .
$$

By $s=i w$, we have

$$
w=-i \frac{v^{*}\left[B_{m}^{*}+z B^{*}(\vec{\varphi})\right]\left[z A_{m}+A(\vec{\varphi})\right] v}{v^{*}\left[B_{m}^{*}+z B^{*}(\vec{\varphi})\right]\left[z B_{m}+B(\vec{\varphi})\right] v} .
$$

For (55), define

$$
\begin{gathered}
M=z A_{m}+A(\vec{\varphi}), \\
N=z B_{m}+B(\vec{\varphi}) .
\end{gathered}
$$

Then

$$
N v v^{*} M^{*}+M v v^{*} N^{*}=0 .
$$

That is,

$$
v^{*} N^{*} N v v^{*} M^{*} N v+v^{*} N^{*} M v v^{*} N^{*} N v=0 .
$$

Because

$$
\begin{array}{r}
v^{*}\left[z B_{m}+B(\vec{\varphi})\right]^{*}\left[z B_{m}+B(\vec{\varphi})\right] v \neq 0, \\
v^{*} N^{*} N v \neq 0,
\end{array}
$$

we have

$$
v^{*} M^{*} N v+v^{*} N^{*} M v=0 .
$$

We have

$$
v^{*} M^{*} N v+\left[v^{*} M^{*} N v\right]^{*}=0 .
$$

So

$$
v^{*} M^{*} N v=v^{*}\left[B_{m}^{*}+z B^{*}(\vec{\varphi})\right]\left[z A_{m}+A(\vec{\varphi})\right] v \in i \mathbb{R} .
$$

So we get that $w \in \mathbb{R}$. Next, by $\varphi_{k}=w \tau_{k}, z=e^{-i w \tau_{m}}$, we can get

$$
\begin{aligned}
\tau_{k} & =\frac{\varphi_{k}+2 n_{k} \pi}{w}, \quad n_{k} \in \mathbb{Z}, k=1,2, \ldots, m-1, \\
\tau_{m} & =\frac{-\operatorname{Arg} z+2 n_{m} \pi}{w}, \quad n_{m} \in \mathbb{Z} .
\end{aligned}
$$

From above conclusion, we find critical delays of the general neutral delay-differential equation with multiple delays. By stable theory, we can also discuss the stability of system (44). 


\section{Conclusion}

In this paper, we consider singular neutral differential systems with multiple delays. By applying algebraic method, such as the matrix pencil spectrum, general eigenvalues, Kronecker product, and linear operators, we discussed eigenvalues and stability of delay-differential systems (1). For the commensurate case, we expatiate the distribution of purely imaginary eigenvalue, the delay-independent, or delay-dependent stability. For the incommensurate case, we also get the critical delays in which stability changed. Certainly, applying algebraic methods to analyze dynamical properties of singular neutral differential systems with delays is a new and immature field, so we believe that algebraic methods used to research the stability of dynamical systems would be more interest in the future.

\section{Conflict of Interests}

The authors declare that there is no conflict of interests regarding the publication of this paper.

\section{Acknowledgments}

The authors acknowledge the National Natural Science Foundations of China (11426058) and the Fundamental Research Funds for the Central Universities (2572015CB25).

\section{References}

[1] J. K. Hale, E. F. Infante, and F. S.-P. Tsen, "Stability in linear delay equations," Journal of Mathematical Analysis and Applications, vol. 105, no. 2, pp. 533-555, 1985.

[2] S. L. Campbell and V. H. Linh, "Stability criteria for differentialalgebraic equations with multiple delays and their numerical solutions," Applied Mathematics and Computation, vol. 208, no. 2, pp. 397-415, 2009.

[3] T. E. Simos, "Closed Newton-Cotes trigonometrically-fitted formulae of high order for long-time integration of orbital problems," Applied Mathematics Letters, vol. 22, no. 10, pp. 16161621, 2009.

[4] W. Jiang, The Degenerate Differential Systems with Delay, Anhui University Press, Hefei, China, 1998.

[5] T. E. Simos, "Exponentially and trigonometrically fitted methods for the solution of the Schrödinger equation," Acta Applicandae Mathematicae, vol. 110, no. 3, pp. 1331-1352, 2010.

[6] W. Zhu and L. R. Petzold, "Asymptotic stability of linear delay differential-algebraic equations and numerical methods," Applied Numerical Mathematics, vol. 24, no. 2-3, pp. 247-264, 1997.

[7] L. Li and Y. Yuan, "Dynamics in three cells with multiple time delays," Nonlinear Analysis: Real World Applications, vol. 9, no. 3, pp. 725-746, 2008.

[8] J. Ma, B. Zheng, and C. Zhang, "A matrix method for determining eigenvalues and stability of singular neutral delaydifferential systems," Journal of Applied Mathematics, vol. 2012, Article ID 749847, 11 pages, 2012.

[9] B. Zheng, J. Ma, H. Zheng, and C. Zhang, "Symmetric bifurcations in a ring of coupled differential equation with piecewise continuous arguments," International Journal of Bifurcation and Chaos, vol. 21, no. 2, pp. 431-436, 2011.

[10] L. P. Shayer and S. A. Campbell, "Stability, bifurcation, and multistability in a system of two coupled neurons with multiple time delays," SIAM Journal on Applied Mathematics, vol. 61, no. 2, pp. 673-700, 2000.

[11] H.-O. Walther, "A periodic solution of a differential equation with state-dependent delay," Journal of Differential Equations, vol. 244, no. 8, pp. 1910-1945, 2008.

[12] D. Q. Cao, P. He, and Y. M. Ge, "Simple algebraic criteria for stability of neutral delay-differential systems," Journal of the Franklin Institute, vol. 342, no. 3, pp. 311-320, 2005.

[13] J. X. Kuang, J. X. Xiang, and H. J. Tian, “The asymptotic stability of one-parameter methods for neutral differential equations," BIT. Numerical Mathematics, vol. 34, no. 3, pp. 400-408, 1994.

[14] E. Fridman, "Stability of linear descriptor systems with delay: a Lyapunov-based approach," Journal of Mathematical Analysis and Applications, vol. 273, no. 1, pp. 24-44, 2002.

[15] H. Zhang and W. Jiang, "Stability of neutral singular differential systems with multiple time-varying delays," Chinese Journal of Engineering Mathematics, vol. 28, no. 2, pp. 265-271, 2011.

[16] C. E. Avellar and J. K. Hale, "On the zeros of exponential polynomials," Journal of Mathematical Analysis and Applications, vol. 73, no. 2, pp. 434-452, 1980.

[17] W. Michiels, K. Engelborghs, D. Roose, and D. Dochain, "Sensitivity to infinitesimal delays in neutral equations," SIAM Journal on Control and Optimization, vol. 40, no. 4, pp. 11341158, 2002.

[18] D. S. Mackey, N. Mackey, C. Mehl, and V. Mehrmann, "Vector spaces of linearizations for matrix polynomials," SIAM Journal on Matrix Analysis and Applications, vol. 28, no. 4, pp. 971-1004, 2006.

[19] J. Louisell, "A matrix method for determining the imaginary axis eigenvalues of a delay system," IEEE Transactions on Automatic Control, vol. 46, no. 12, pp. 2008-2012, 2001.

[20] W. Michiels and T. Vyhlídal, "An eigenvalue based approach for the stabilization of linear time-delay systems of neutral type," Automatica, vol. 41, no. 6, pp. 991-998, 2005.

[21] E. Jarlebring, "On critical delays for linear neutral delay systems," in Proceedings of the European Control Conference, 2007. 


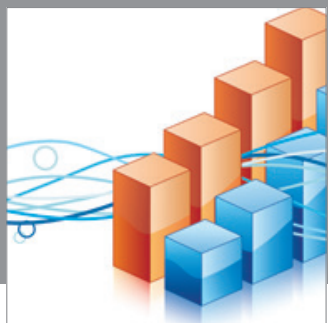

Advances in

Operations Research

mansans

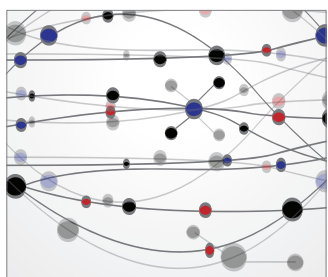

The Scientific World Journal
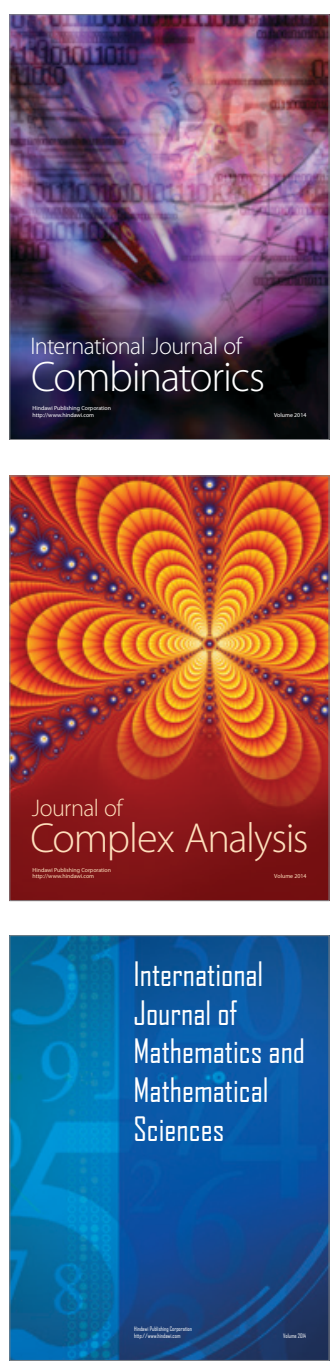
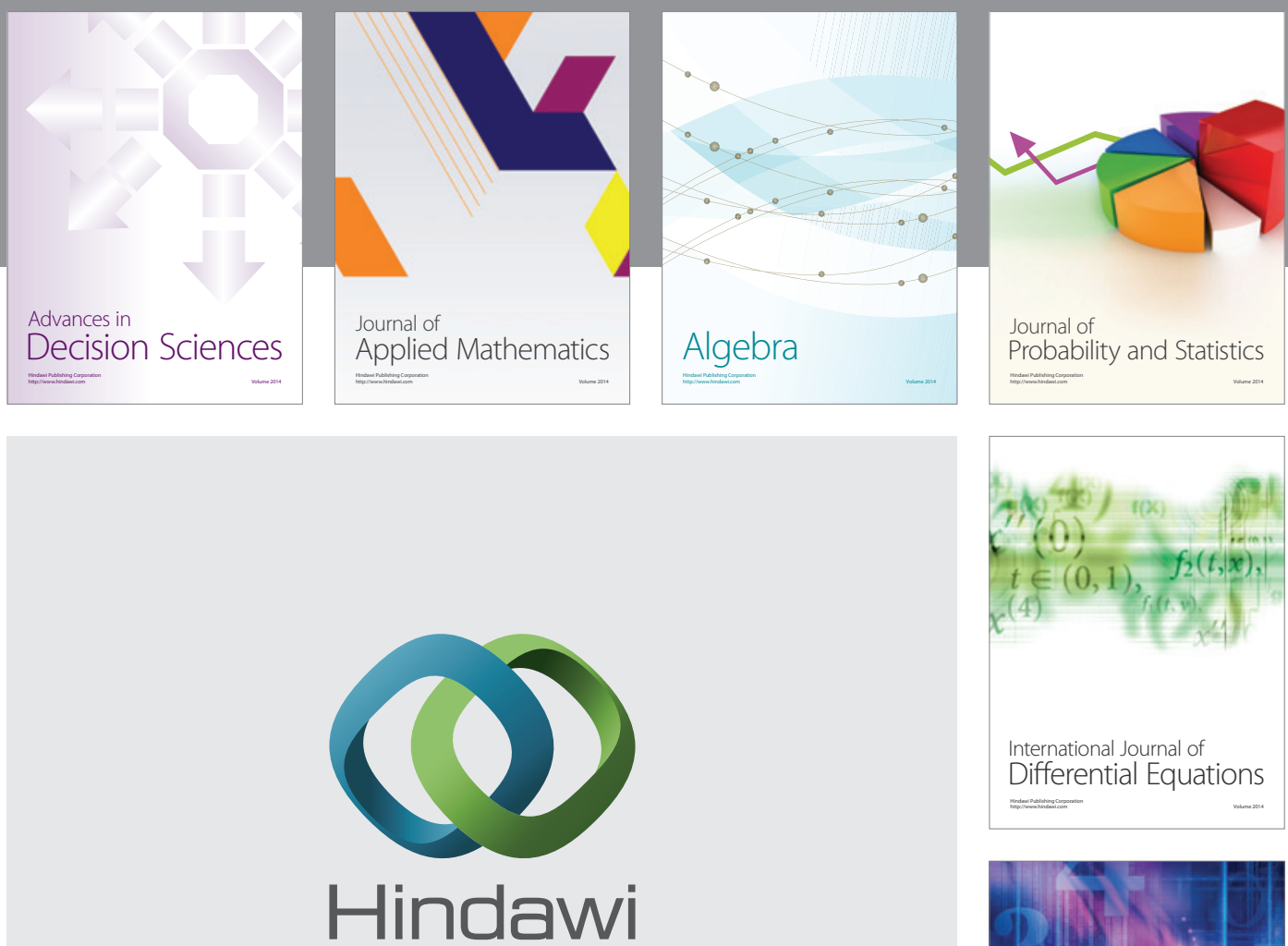

Submit your manuscripts at http://www.hindawi.com
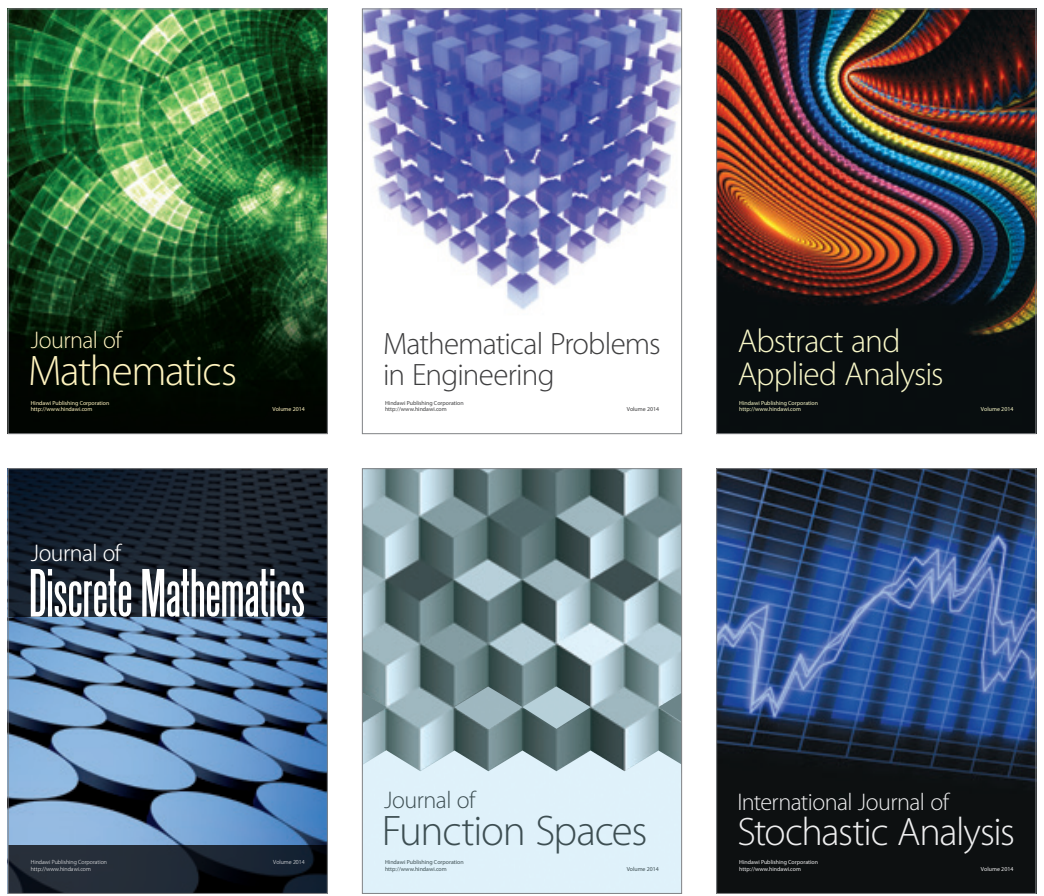

Journal of

Function Spaces

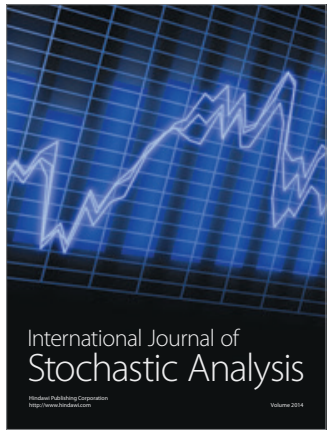

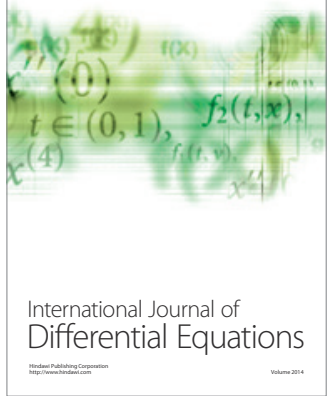
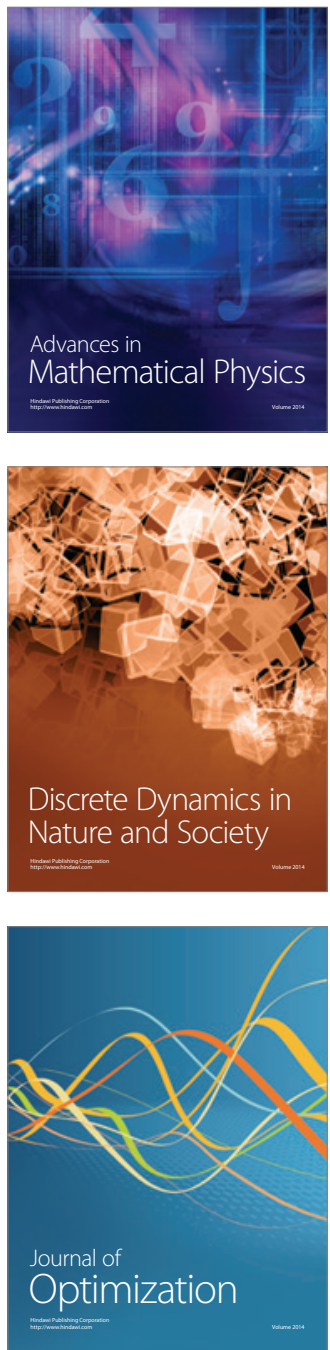